\title{
Catálogo dos Peixes Marinhos do Laboratório de Ictiologia da Universidade Federal do Rio de Janeiro. Partel: Chondrichthyes (Rajiformes). Teleostei (Elopiformes a Dactylopteriformes).
}

\author{
Paulo Roberto Duarte Lopes ${ }^{1}$
}

\begin{abstract}
The present paper is the first part of the catalogue of marine fishes belonging to the collection of the Laboratory of Ichthyology (Universidade Federal do Rio de Ianeiro-RJ) to be published. Here are included Chondrichthyes (Rajiformes) and part of Osteichthyes, Teleostei (Elopiformes to Dactylopteriformes) in a total amount of 30 families, 52 genera and 62 species.

The most part of the material have been collected at the littoral of the State of Rio de Janeiro. For each species considered is given the vulgar name (when known in Southeastern Brazil), the known distribution (in Western Atlantic Ocean) and some data as collecting locality, total number of collected specimens and the register number in the collection. Scorpaena isthmensis Meek \& Hildebrand, 1928 (Scorpaenidae) is for the first time mentioned for the Southern littoral of Brazil.
\end{abstract}

A coleção de peixes marinhos do Laboratório de Ictiologia (Departamento de Zoologia) da Universidade Federal do Rio de Janeiro (UFRJ) está composta por espécimes coletados principalmente na Baía de Guanabara e na região sob influência da Central Nuclear Almirante Álvaro Alberto, no litoral do Estado do Rio de Janeiro (região sudeste do Brasil).

Nesta primeira parte, que totaliza 30 familias, 52 gêneros e 62 espécies, são incluídas a Classe Chondrichthyes (Ordem Rajiformes com 4 familias e 7 espécies) e parte da Classe Osteichthyes (Elopiformes a Dactylopteriformes)com 26 famılia e 55 espécies.

O material estudado, perfazendo um total de 460 exemplares, foi coletado entre o município de Prado (Estado da Bahia), Brasil e o município de Florianópolis (Estado de Santa Catarina), Brasil.

Scorpaena isthmensis Meek \& Hildebrand, 1928 (Scorpaenidae) teve ampliada a sua área de distribuição geográfica (em seu limite sul, passando de Santos-SP, Brasil para Florianópolis-SC).

${ }^{1}$ Universidade Estadual de Feira de Santana, Departamento de Ciências Biológicas, Campus Universitário, km 3 da BR 116,44061 Feira de Santana - BA. 
Para cada espécie citada neste catálogo são fornecidos: o nome vulgar (quando conhecido, para algumas localidades do sudeste brasileiro), a distribuição conhecida no Atlântico Ocidental (segundo a bibliografia consultada), a procedência do material examinado, a quantidade de exemplares coletados em cada uma dessas localidades e o seu respectivo número de registro no livro da coleção do Laboratório de Ictiologia da UFRJ .

\section{RESULTADOS}

Classe Chondrichthyes

Subclasse Elasmobranchii

Ordem Rajiformes

\section{Fam1lia Rhinobatidae}

\subsection{Rhinobatos Linck, 1790}

1.1.1. Rhinobatos percellens (Walbaum, 1792)

Nome vulgar: raia-viola.

Distribuição conhecida: do Caribe à Cananéia (SP).

Material colecionado: Mombaça, Angra dos Reis (RJ) - 1 exemplar - 770204

Saco da Piraquara de Fora, Angra dos Reis (RJ) - 1 exemplar - 830520

\subsection{Zapteryx Jordan \& Gilbert, 1880}

1.2.1.Zapteryx brevirostris (Muller \& Henle, 1841)

Distribuição conhecida: de Cabo Frio (RJ) à Argentina (Mar del Plata).

Material colecionado: $23^{\circ} 02^{\prime}$ lat. S, $43^{\circ} 17^{\prime}$ long. W, Il has Tijucas (RJ) - 1 exemplar -770314

Ilha Grande (RJ) - 1 exemplar - 840578

O exemplar 770314 foi coletado a uma profundidade de 38 metros.

Apesar de FIGUEIREDO (1981) considerar necessário erigir um novo gênero para alojar essa espécie, optou-se pela sua manutenção em Zapteryx Jordan \& Gilbert, 1880 até que um posicionamento sistemático definitivo seja estabelecido.

\section{Famılia Rajidae}

2.1. Psammobatis Günther, 1870

2.1.1. Psammobatis glansdissimilis McEachran, 1983

Distribuição conhecida: do Rio de Janeiro, BR ao norte da Argentina.

Material colecionado: $22^{\circ} 59^{\prime}$ lat. S, $42^{\circ} 19^{\prime}$ long. W, Araruama (RJ) - 1 exemplar - 860776, coletado a uma profundidade de 50 metros.

2.2 Raja Linnaeus, 1758

2.2.1. Raja cyclophora Regan, 1903

Nome vulgar: raia-santa .

Distribuição conhecida: de Cabo Frio (RJ) à Argentina (Puerto Quequén).

Material colecionado: $23^{\circ} 02^{\prime}$ lat. S, $43^{\circ} 17^{\prime}$ long. W, Ilhas Tijucas (RJ) - 1 exemplar - 840566, coletado a uma profundidade de 38 metros. 
Vol. 6(2), 1989

\section{Fam1lia Narcinidae}

\subsection{Narcine Henle, 1834}

3.1.1. Narcine brasiliensis (Olfers, 1831)

Nome vulgar: treme-treme, raia-elétrica.

Distribuição conhecida: dos EUA (Carolina do Norte) ao norte da Argentina.

Material colecionado: Mombaça, Angra dos Reis (RJ) - 1 exemplar - 770197

Base Oceanográfica Norte da Universidade de São Paulo, Ubatuba (SP) - 1 exemplar - 790346

\section{Fam1lia Gymnuridae}

4.1. Gymnura Van Hasselt, 1823

4.1.1. Gymnura altavela (Linnaeus, 1758)

Nome vulgar: raia-manteiga, raia-borboleta.

Distribuição conhecida: dos EUA (Massachusetts) à Argentina .

Material colecionado: Baía de Guanabara (RJ) - 2 exemplares - 860775

4.1.2. Gymnura micrura (Bloch \& Schneider, 1801)

Nome vulgar: raia-manteiga, raia-borboleta .

Distribuição conhecida: dos EUA (Nova Inglaterra) ao sul do Brasil $\left(32^{\circ} 17^{\prime}\right.$ lat. S, $51^{\circ} 31^{\prime}$ long. W).

Material colecionado: Cumuruxatiba, Prado (BA) - 1 exemplar - 760054

O limite sul da distribuição desta espécie foi fornecido por Sadowsky (comunicação pessoal).

Classe Osteichthyes

Subclasse Actinopterygii

Infraclasse Teleostei

Ordem Elopiformes

\section{Fam11ia Elopidae}

\subsection{Elops Linnaeus, 1766}

\subsubsection{Elops saurus Linnaeus, 1766}

Nome vulgar: ubarama.

Distribuição conhecida: dos EUA (Nova Inglaterra) ao sudeste do Brasil.

Material colecionado: Lagoa de Maricá, Maricá (RJ) - 1 exemplar - 860741

Baía de Guanabara (RJ) - 1 exemplar - 860748

\section{Fam1lia Albulidae}

\subsection{Albula Scopoli, 1777}

6.1.1. Albula vulpes (Linnaeus, 1758)

Nome vulgar: ubarana-focinho-de-rato.

Distribuição conhecida: dos EUA (Massachusetts) à Porto Belo (SC).

Material colecionado: Praia da Guanabara, Ilha do Governador, Baía de Guanabara (RJ) - 1 exemplar - 870920

Ordem Anguilliformes 


\section{Familia Muraenidae}

\subsection{Gymnothorax Bloch, 1794}

\subsubsection{Gymnothorax ocellatus Agassiz, 1831}

Nome vulgar: moréia-pintada.

Distribuição conhecida: das Grandes Antilhas ao sul do Brasil.

Material colecionado: Meaípe, Guarapari (ES) - 1 exemplar - 820371; Ao largo da Ilha dos Franceses, Cabo Frio (RJ) - 1 exemplar - 860774; Do meio da Baía de Guanabara (RJ) - 2 exemplares - 870885; Ilha Rasa (RJ) - 3 exemplares 770284; Itacurussá, Mangaratiba (RJ) - 3 exemplares - 840576; Ilha Grande (RJ) - 6 exemplares - $770307 ; 23^{\circ} 12^{\prime}$ lat. S, $44^{\circ} 07^{\prime}$ long. W - $23^{\circ} 13^{\prime}$ lat. S, 44 11 ' long. W, Ilha Grande (RJ) - 2 exemplares- 770294; Saco da Piraquara de Fora, Angra dos Reis (RJ) - 1 exemplar - 830507.

O exemplar 860774 foi coletado a uma profundidade de 35 metros.

$\mathrm{Na}$ identificação desta espécie seguiu se o critério adotado por FIGUEIREDO \& MENEZES (1978).

\subsection{Lycodontis Mc Clelland, 1844}

7.2.1. Lycodontis funebris Ranzani, 1840

Nome vulgar: moréia, caramuru .

Distribuição conhecida: ocorre no Atlântico Ocidental tropical.

Material colecionado: Cumuruxatiba, Prado (BA) - 1 exemplar - 760027.

7.2.2. Lycodontis vicinus (Castelnau, 1855)

Nome vulgar: moréia.

Distribuição conhecida: das Bermudas ao sudeste do Brasil.

Material colecionado: Cumuruxatiba, Prado (BA) - 1 exemplar - 760020.

7.3. Muraena Linnaeus, 1758

\subsubsection{Muraena sp}

Material colecionado: Ilha Rasa (RJ) - 1 exemplar - 770285

A única espécie do gênero no litoral sudeste do Brasil, segundo FIGUEIREDO \& MENEZES (1978), é $M$. miliaris (Kaup, 1856). O exemplar acima citado difere da diagnose apresentada por BOHLKE (1978) para M. miliaris (Kaup, 1856) nos seguintes aspectos: o colorido do corpo é constituído por manchas brancas arredondadas, cuja densidade diminui em direção à cauda, sobre fundo marrom e que são maiores na maxila inferior, onde tendem a fundirem se (apesar da coloração de $M$. miliaris (Kaup, 1856) ser altamente variável, o tipo apresentado não é relatado para esta espécie); presença de uma única série de dentes na porção anterior da maxila inferior (em M. miliaris (Kaup, 1856) são citadas duas séries) e presença de uma única série de dentes na maxila superior(em M. miliaris (Kaup, 1856) são citadas duas ou três séries).

\section{Famılia Congridae}

\subsection{Ariosoma Swainson, 1838}

\subsubsection{Ariosoma sp}

Material largo da Ilha dos Franceses, Cabo Frio (RJ) - 2 exemplares - 860783; Ao largo do litoral de Araruama (RJ) - 2 exemplares - 860779, 860781; Ilha Grande (RJ) - 2 exemplares - $770306 ; 23^{\circ} 12^{\prime}$ lat. S, $44^{\circ} 07^{\prime}$ long, W - $23^{\circ} 13^{\prime}$ lat. S, $44^{\circ} 11^{\prime}$ long. W, Ilha Grande (RJ) - 1 exemplar - 770278 .

O exemplar 860779 está representado apenas pela cabeça e parte das nadadeiras 
peitorais, tendo sido coletado a uma profundidade de 46 metros. Os exemplares 860781 e 860783 estão representados apenas pela cabeça e parte do corpo, tendo sido coletados em profundidades de 40 e 35 metros, respectivamente.

FIGUEIREDO \& MENEZES (1978) identificaram o material pertencente à este gênero para o sudeste do Brasil como A. opisthophthalma (Ranzani, 1838). Contudo, posteriormente, FIGUEIREDO (1981) observou diferenças que levaram no a caracterizar os exemplares anteriormente examinados como sendo duas espécies as quais, por não ter sido possível identificação a nível específico, foram denominadas Ariosoma sp A e Arisoma sp B. Sendo assim, optou -se pela identificação apenas a nível genérico, pois ainda faltam muitas dúvidas a serem dissipadas no que se refere à correta identidade dos exemplares conhecidos para o su deste do Brasil (Figueiredo, comunicação pessoal).

\section{Familia Ophichthidae}

\subsection{Ahlia Jordan \& Davis, 1892}

\subsubsection{Ahlia egmontis (Jordan, 1884)}

Distribuição conhecida: dos EUA (Flórida) ao sudeste do Brasil .

Material colecionado: Praia Vermelha, Baía de Guanabara (RJ) - 1 exemplar 840606.

9.2. Ophichthus Ahl, 1789

\subsubsection{Ophichthus gomesii (Castelnau, 1855)}

Distribuição conhecida: dos EUA (Nova Inglaterra) à Argentina .

Material colecionado: Ao largo da Ilha dos Franceses, Cabo Frio (RJ) - 2 exemplares - 860782, 860784; Ao largo do litoral de Araruama (RJ) - 1 exemplar 860780; Do meio da Baía de Guanabara (RJ) - 2 exemplares - 860869; Praia da Urca, Baía de Guanabara (RJ) - 1 exemplar - 790356; Ilha Grande (RJ) 1 exemplar - 840672 .

O exemplar 860780 foi coletado a uma profundidade de 46 metros. Os exemplares 860872 e 860874 estão representados apenas pela cabeça e parte do corpo, tendo sido coletados a uma profundidade de 35 metros.

\section{Ordem Clupeiformes}

\section{Familia Clupeidae}

\subsection{Brevoortia Gill, 1861}

10.1.1.Brevoortia aurea (Spix, 1829)

Nome vulgar: savelha.

Distribuição conhecida: da Bahia, BR a São Paulo, BR.

Material colecionado: Lagoa da Barra, Maricá (RJ) - 8 exemplares - 850710; Rio Mombuca, Maricá (RJ) - 7 exemplares - 820362; Lagoa de Maricá, Maricá (RJ) - 14 exemplares - 850712,850713, 850731; Ilhas das Palmas, Baía de Gua nabara $(\mathrm{RJ})$ - 1 exemplar - 840634 .

$\mathrm{Na}$ identificação desta espécie seguiu -se o critério adotado por FIGUEIREDO \& MENEZES (1978).

10.2. Chirocentrodon Günther, 1860

10.2.1. Chirocentrodon bleekerianus (Poey, 1867)

Distribuição conhecida: do Canal de Iucatã à Torres (RS). 
Material colecionado: Do meio da Baía de Guanabara (RJ) - 4 exemplares860865, 860876; Em águas adjacentes à Central Nuclear Almirante Álvaro Alberto, Angra dos Reis (RJ) - 5 exemplares - 830476.

10.3. Harengula Valenciennes, 1847

10.3.1. Harengula clupeola (Cuvier, 1829)

Nome vulgar: sardinha-cascuda .

Distribuição conhecida: dos EUA (Flórida) à São Paulo, BR.

Material colecionado: Praia da Boa Viagem, Niterói (RJ) - 2 exemplares - 760083; Baía de Guanabara (RJ) - 5 exemplares - 860747; Do meio da Baía de Guanabara (RJ) - 1 exemplar - 840633; Praia de Guanabara, Ilha do Governador, Baía de Guanabara (RJ) - 23 exemplares - 860801; Praia do Barão, Ilha do Governador, Baía de Guanabara (RJ) - 1 exemplar - 860735; Praia do Leme, Rio de Janeiro (RJ) - 1 exemplar - 760004; Praia de Copacabana, Rio de Janeiro (RJ) - 2 exemplares - 760096; Em águas adjacentes à Central Nuclear Almirante Álvaro Alberto, Angra dos Reis (RJ) - 6 exemplares - 830475; Saco da Piraquara de Fora, Angra dos Reis (RJ) - 6 exemplares - 830420, 830443, 830459, 830505, 830531; Ca ieira da Barra Sul, Florianópolis (SC) - 1 exemplar - 770227.

$\mathrm{Na}$ identificação desta espécie seguiu-se o criterio adotado por FIGUEIREDO \& MENEZES (1978).

\subsection{Opisthonema Gill, 1861}

10.4.1. Opisthonema oglinum (Le Sueur, 1817)

Nome vulgar: sardinha-bandeira.

Distribuição conhecida: dos EUA (Nova Inglaterra) à Argentina .

Material colecionado: Canal de Ponta Negra, Maricá (RJ) - 3 exemplares 860841, 870897; Ilha Rasa, Baía de Guanabara (RJ) - 1 exemplar - 840602; Ponta da Pombeba, Rio de Janeiro (RJ) - 1 exemplar - 760105; Mombaça, Angra dos Reis (RJ) - 1 exemplar - 770211; Saco da Piraquara de Dentro, Angra dos Reis (RJ) - 1 exemplar - 830439.

10.5.Pellona Valenciennes

10.5.1. Pellona harroweri (Fowler, 1917)

Nome vulgar: sardinha-prata.

Distribuição conhecida: do Panamá ao Rio Grande do Sul, BR.

Material colecionado: Saco da Piraquara de Fora, Angra dos Reis (RJ) - 2 exemplares - 830435, 830532 .

\subsection{Sardinella Valenciennes}

10.6.1. Sardinella brasiliensis (Steindachner, 1789)

Nome vulgar: sardinha, sardinha-verdadeira.

Distribuição conhecida: do Rio de Janeiro, BR para o sul.

Material colecionado: Praia da Boa Viagem, Niterói (RJ) - 4 exemplares - 840631; do meio da Baia de Guanabara (RJ) - 3 exemplares - 840609; Em águas adjacentes à Central Nuclear Almirante Álvaro Alberto, Angra dos Reis (RJ) - 2 exemplares - 830488; Saco da Piraquara de Fora, Angra dos Reis (RJ) - 1 exemplar 830430; Caieira da Barra Sul, Florianópolis (SC) - 1 exemplar - 770288.

O exemplar 770288 foi retirado do estômago de um Trichiurus lepturus (Perciformes, Trichiuridae).

Na identificação desta espécie seguiu-se o critério adotado por FIGUEIREDO \& MENEZES (1978) 
Vol. 6(2), 1989

\section{Familia Engraulidae}

\subsection{Anchoa Jordan \& Evermann, 1902}

11.1.2. Anchoa jamuaria (Steindachner, 1879)

Distribuição conhecida: da Venezuela ao Rio Grande do Sul, BR.

Material colecionado: Ba!a de Guanabara (RJ) - 1 exemplar - 860788; Ilha do

Catalão, Baìa de Guanabara (RJ) - 1 exemplar - 860739.

11.1.3. Anchoa lyolepis (Evermann \& Marsh, 1902)

Distribuição conhecida: dos EUA (Cabo Hatteras) à Rio Grande (RS).

Material colecionado: Praia da Boa Viagem, Niterói (RJ) - 3 exemplares - 760078.

11.1.4. Anchoa spinifera (Valenciennes, 1848)

Nome vulgar: sardinha-boca-de-cobra.

Distribuição conhecida: do Panamá à Porto Belo (SC).

Material colecionado: Cumuruxatiba, Prado (BA) - 1 exemplar - 760031.

11.1.5. Anchoa tricolor (Agassiz, 1829)

Distribuição conhecida: do Ceará, BR à Argentina.

Material colecionado: Baía de Guanabara (RJ) - 18 exemplares - 860745; Praia de Guanabara, Ilha do Governador, Baĭa de Guanabara (RJ) - 10 exemplares 860740, 860874, 870902.

\subsection{Cetengraulis Günther, 1868}

\subsubsection{Cetengraulis edentulus (Cuvier, 1828)}

Distribuição conhecida: no Caribe e do Panamá à Santa Catarina, BR.

Material colecionado: Do meio da Baía de Guanabara (RJ) - 1 exemplar - 840624; Praia da Guanabara, Ilha do Governador, Baía de Guanabara (RJ) - 17 exemplares - 860836, 870894; Praia do Barão, Ilha do Governador, Baĭa de Guanabara (RJ) - 7 exemplares - 860738; Ilha do Catalão, Baĩa de Guanabara (RJ) - 6 exemplares - 840586; Sepetiba, Rio de Janeiro (RJ) - 1 exemplar - 830405; Saco da Piraquara de Dentro, Angra dos Reis (RJ) - 3 exemplares - 830429.

11.3. Engraulis Cuvier, 1817.

11.3.1. Engraulis anchoita Hubbs \& Marini, 1935

Distribuição conhecida: do norte do Rio de Janeiro, BR à Argentina.

Material colecionado: Ilha Grande (RJ) - 1 exemplar - 840664.

11.4. Lycengraulis Günther, 1868

11.4.1. Lycengraulis grossidens (Agassiz, 1829)

Nome vulgar: manjubão.

Distribuição conhecida: da Venezuela à Argentina .

Material colecionado: Ilha da Marambaia (RJ) - 1 exemplar - 760106.

\section{Ordem Siluriformes}

\section{Familia Ariidae}

\subsection{Bagre Oken, 1817}

\subsubsection{Bagre bagre (Linnaeus, 1766)}

Nome vulgar: Bagre-bandeira

Distribuição conhecida: da Colômbia a Florianópolis (SC).

Material colecionado: Sepetiba, Rio de Janeiro (RJ) - 1 exemplar - 830414.

12.1.2. Bagre marinus (Mitchill, 1815) 
Nome vulgar: bagre-bandeira.

Distribuição conhecida: dos EUA (Carolina do Norte) ao sudeste do Brasil. Material colecionado: Cumuruxatiba, Prado (BA) - 1 exemplar - 760019.

12.2. Cathorops Jordan \& Gilbert, 1883

12.2.1. Cathorops spixii (Agassiz, 1829)

Nome vulgar: bagre-amarelo, bagre-mandi.

Distribuição conhecida: da Venezuela ao sudeste do Brasil.

Material colecionado: Lagoa de Maricá, Maricá (RJ) - 2 exemplares - 850717, 850718; Base Oceanográfica Norte da Universidade de São Paulo, Ubatuba (SP) 1 exemplar - 790345 .

$\mathrm{Na}$ identificação desta espécie seguiu-se o critério adotado por TAYLOR \& MENEZES (1978).

12.3. Genidens Castelnau, 1855.

12.3.1. Genidens genidens (Valenciennes, 1839)

Nome vulgar: bagre-urutu, bagre-veludo.

Distribuição conhecida: das Guianas ao Rio da Prata.

Material colecionado: Lagoa de Maricá, Maricá (RJ) - 5 exemplares - 850716; Baía de Guanabara (RJ) - 1 exemplar - 860746; Ilha do Catalão, Baĭa de Guanabara (RJ) - 1 exemplar - 840629; Praia do Catalão, Ilha do Fundão, Baia de Guanabara (RJ) - 1 exemplar - 760110.

Sepetiba, Rio de Janeiro (RJ) - 1 exemplar - 830413.

12.4. Netuma Bleecker, 1858.

12.4.1. Netuma barba (Lacépède, 1803)

Nome vulgar: bagre-branco.

Distribuição conhecida: do sudeste do Brasil ao Rio da Prata.

Material colecionado: Lagoa de Maricá, Maricá (RJ) - 1 exemplar - 860763; Do meio da Baía de Guanabara (RJ) - 1 exemplar - 860734; Sepetiba, Rio de Janeiro (RJ) - 3 exemplares - 830412 .

12.5. Sciadeichthys Bleeker, 1858

12.5.1. Sciadeichthys luniscutis (Valenciennes, 1840)

Nome vulgar: bagre.

Distribuição conhecida: das Guianas ao sudeste do Brasil.

Material colecionado: Base Oceanográfica Norte da Universidade de São Paulo, Ubatuba (SP) - 1 exemplar - 790347.

\section{Ordem Myctophiformes}

\section{Familia Synodontidae}

13.1. Synodus Gronow, 1763.

13.1.1. Synodus foetens (Linnaeus, 1766)

Nome vulgar: peixe-lagarto.

Distribuição conhecida: dos EUA (Nova Inglaterra) à Tramandaĭ (RS).

Material colecionado: Do meio da Baía de Guanabara (RJ) - totalizando 2 exemplares - 840635, 840677; Praia da Guanabara, Ilha do Governador, Baia de Guanabara (RJ) - 2 exemplares - 840601, 840657; Saco da Piraquara de Fora, Angra dos Reis (RJ) - 6 exemplares - 830502, 830525 . 
Vol. 6(2), 1989

13.2. Trachinocephalus Gill, 1861.

13.2.1. Trachinocephalus myops (Forster, 1861)

Nome vulgar: peixe-lagarto.

Distribuição conhecida: do Canadá (Nova Escócia) à Santa Catarina, BR.

Material colecionado: $23^{\circ} 02^{\prime}$ lat. S, $43^{\circ} 17^{\prime}$ long. W, Ilhas Tijucas (RJ) - 1 exemplar - 770313; Ilha Grande (RJ) - 1 exemplar - 770300; Em águas adjacentes à Central Nuclear Almirante Álvaro Alberto, Angra dos Reis (RJ) - 1 exemplar 830460 .

O exemplar 770313 foi coletado a uma profundidade de 38 metros.

\section{Ordem Ophidiiformes}

\section{Familia O phidiidae}

14.1. Ophidion Linnaeus, 1758

14.1.1. Ophidion holbrooki (Putnam, 1874)

Distribuição conhecida: no Golfo do México, Venezuela e do Rio de Janeiro, BR à Santa Catarina, BR.

Material colecionado: $23^{\circ} 12^{\prime}$ lat. S, $44^{\circ} 07^{\prime}$ long. W - $23^{\circ} 13^{\prime}$ lat. S, $44^{\circ} 11^{\prime}$ long. W, Ilha Grande (RJ) - 4 exemplares - 770285.

Na identificação desta espécie seguiu-se o critério adotado por FIGUEIREDO \& MENEZES (1978).

14.2. Raneya Robins, 1961.

14.2.1. Raneya fluminensis (Ribeiro, 1903)

Distribuição conhecida: do Rio de Janeiro, BR à Argentina (Puerto Quequén). Material colecionado: Ilha Grande (RJ) - 1 exemplar - 840569.

\section{Ordem Gadiformes}

\section{Familia Gadidae}

15.1. Urophycis Gill, 1864.

15.1.1. Urophycis brasiliensis (Kaup, 1858)

Nome vulgar: abrótea.

Distribuição conhecida: do Rio de Janeiro, BR à Argentina (Mar del Plata). Material colecionado: $23^{\circ} 12^{\prime}$ lat. S, $44^{\circ} 07^{\prime}$ long. W - $23^{\circ} 13^{\prime}$ lat. S, $44^{\circ} 11^{\prime}$ 'long. W, Ilha Grande (RJ) - 2 exemplares - 770290.

\section{Familia Merlucciidae}

16.1. Merluccius Rafinesque, 1810.

16.1.1. Merluccius hubbsi Marini, 1933.

Nome vulgar: merluza.

Distribuição conhecida: de Cabo Frio (RJ) à Argentina.

Material colecinado: $23^{\circ} 02^{\prime}$ lat. S, $43^{\circ} 17^{\prime}$ long. W, Ilhas Tijucas (RJ) - 2 exemplares - 770312; Ilha Grande (RJ) - 3 exemplares - 840668.

Os exemplares 770312 foram coletados a uma profundidade de 38 metros. 
Ordem Batrachoidiformes

\section{Familia Batrachoididae}

17.1. Porichthys Girard, 1855

17.1.1. Porichthys porosissimus (Valenciennes, 1837)

Nome vulgar: mamangá-liso, mangangá-liso.

Distribuição conhecida: de Vitória (ES) à Argentina.

Material colecionado: Rio das Ostras, Casimiro de Abreu (RJ) - 1 exemplar 760122; Ao largo da Ilha dos Franceses, Cabo Frio (RJ) - 1 exemplar 860778; Do meio da Baía de Guanabara (RJ) - 1 exemplar - 860818; Ilha Grande (RJ) - 8 exemplares - $770304 ; 23^{\circ} 12^{\prime}$ lat. S, $44^{\circ} 07^{\prime}$ long. W - $23^{\circ} 13^{\prime}$ lat. S, $44^{\circ} 11^{\prime}$ long. W, Ilha Grande (RJ) - 2 exemplares - 770283.

O exemplar 860778 foi coletado a uma profundidade de 35 metros.

$\mathrm{Na}$ identificação desta espécie seguiu-se o critério adotado por MENNI \& MIQUELARENA (1976) que considera $P$. porosissimus (Valenciennes, 1837) e $P$. plectrodon Goode \& Bean, 1882 como duas espécies distintas, tendo por base a morfologia do otólito. Já GILBERT in FIGUEIREDO (1981), pelo estudo da morfologia externa, considera $P$. plectrodon Goode \& Bean, 1882, que ocorre dos EUA (Virglnia) até pelo menos o Suriname, como sinônima de $P$. porosissimus (Valenciennes, 1837). Esse último critério, seguido também por outros autores, tem causado dificuldades no que se refere à determinação do limite norte de distribuição de $P$. porosissimus (Valenciennes, 1837), considerado ainda como provisório (FIGUEIREDO \& MENEZES, 1978).

\section{Ordem Lophiiformes}

\section{Familia Lophiidae}

\subsection{Lophius Linnaeus, 1758}

18.1.1. Lophius gastrophysus Ribeiro, 1915.

Nome vulgar: peixe-diabo.

Distribuição conhecida: dos EUA (Cabo Hatteras) ao norte da Argentina.

Material colecionado: Ilha Grande (RJ) - 4 exemplares - 840670.

Um exemplar de Dules auriga (Perciformes, Serranidae), com $108 \mathrm{~mm}$ de comprimento total, foi retirado da boca de um dos espécimes acima citados.

\section{Fanilia Antennariidae}

\subsection{Phrynelox Whitley, 1931}

19.1.1. Phrynelox scaber (Cuvier, 1817)

Nome vulgar: peixe-sapo.

Distribuição conhecida: dos EUA (Nova Jérsei) ao sul do Brasil.

Material colecionado: $23^{\circ} 02^{\prime}$ lat. S, $43^{\circ} 17^{\prime}$ long. W, Ilhas Tijucas (RJ) - 1 exemplar - 770310; Ilha Grande (RJ) - 2 exemplares - 840662.

O exemplar 770310 foi coletado a uma profundidade de 38 metros.

\section{Familia Ogcocephalidae}

20.1.Ogcocephalus Fischer, 1813 
Vol. 6(2), 1989

20.1.1. Ogcocephalus vespertilio (Linnaeus, 1758)

Nome vulgar: peixe-morcego.

Distribuição conhecida: dos EUA (Nova Iorque) ao Uruguai.

Material colecionado: Meaïpe, Guarapari- ES - 1 exemplar - 760063; 22 ${ }^{\circ} 59^{\prime}$ lat. S, $42^{\circ} 19^{\prime}$ long. W, Araruama (RJ) - 1 exemplar - 860777; Praia da Urca, Bala de Guanabara (RJ) - 1 exemplar - 870921; Ilhas Tijucas (RJ) - 1 exemplar 760182; Ilha Grande (RJ) - 8 exemplares - 840666.

O exemplar 860777 foi coletado a uma profundidade de 50 metros.

\section{Ordem Gobiesociformes}

\section{Familia Gobiesocidae}

21.1. Gobiesox Lacépède, 1800

21.1.1. Gobiesox strumosus Cope, 1870

Distribuição conhecida: dos EUA (Nova Jérsei) ao Rio Grande do Sul, BR.

Material colecionado: Praia de Itaipu, Niterói (RJ) - 1 exemplar - 760152; Praia da Boa Viagem, Niterói(RJ) - 8 exemplares - 760109, 760112, 780315, 820375, 840567, 860750; Praia de Guanabara, Ilha do Governador, Baía de Guanabara (RJ) - 2 exemplares - 850730; Ilha do Catalão, Baĭa de Guanabara (RJ) - 1 exemplar - 840568; Praia da Urca, Baǐa de Guanabara (RJ) - 2 exemplares - 840616, 860843; Itacurussá, Mangaratiba (RJ) - 1 exemplar - 760082; Ilha da Marambaia (RJ) - 1 exemplar - 760098.

\section{Ordem Atheriniformes}

\section{Familia Exocoetidae}

22.1. Hyporhamphus Gill, 1859

22.1.1. Hyporhamphus unifasciatus (Ranzani, 1842)

Nome vulgar: agulha, panaguaiú.

Distribuição conhecida: das Bermudas ao Uruguai.

Material colecionado: Lagoa de Juparanã, Linhares (ES) - 1 exemplar - 760118; Praia de Guanabara, Ilha do Governador, Baía de Guanabara (RJ) - 4 exemplares - 870887; Pedra de Guaratiba, Rio de Janeiro (RJ) - 1 exemplar - 760093; Praia do Calabouço, Ilha Jaguanum (RJ) - 1 exemplar - 790352.

\section{Familia Belonidae}

\subsection{Strongylura Van Hasselt, 1823}

23.1.1. Strongylura timucu (Walbaum, 1792)

Nome vulgar: agulha.

Distribuição conhecida: dos EUA (Flórida) ao sul do Brasil.

Material colecionado: Barra do Rio Caí, Cumuruxatiba, Prado (BA) - 2 exemplares - 760046, 760048; Meaipe, Guarapari (ES) - 1 exemplar - 820372; Praia da Guanabara, Ilha do Governador, Baĭa de Guanabara (RJ) - 1 exemplar 870905 .

\section{Familia Atherinidae}

24.1. Xenomelaniris Schultz, 1948 
24.1.1. Xenomelaniris brasiliensis (Quoy \& Gaimard, 1824)

Nome vulgar: peixe-rei.

Distribuição conhecida: da Venezuela ao Rio Grande do Sul, BR.

Material colecionado: Barra do Rio Caí, Cumuruxatiba, Prado (BA) - 1 exemplar - 760044; Lagoa de Maricá, Maricá (RJ) - 1 exemplar - 850715; Canal afluente da Lagoa de Itaipu, Niterói (RJ) - 1 exemplar - 760175 ; Praia da Guanabara, Ilha do Governador, Baía de Guanabara (RJ) - 22 exemplares - 840652, 860799; Ilha do Catalão, Baía de Guanabara (RJ) - 17 exemplares - 840583; Sepetiba, Rio de Janeiro (RJ) - 6 exemplares - 790353.

Na identificação desta espécie seguiu-se o critério adotado por FIGUEIREDO \& MENEZES (1978).

\section{Ordem Beryciformes}

\section{Familia Holocentridae}

25.1. Corniger Agassiz, 1829

25.1.1. Corniger spinosus Agassiz, 1829

Nome vulgar: talhão.

Distribuição conhecida: dos EUA (Carolina do Sul) ao Rio de Janeiro, BR.

Material colecionado: Ilha de Maricá, Maricá (RJ) - 1 exemplar - 860810.

25.2. Holocentrus Gronow, 1763

25.2.1. Holocentrus ascensionis (Osbeck, 1765)

Nome vulgar: jaguareçá.

Distribuição conhecida: dos EUA (Carolina do Norte) à Garopaba (SC).

Material colecionado: Meaïpe, Guarapari (ES) - 4 exemplares - 760002, 760057 , 760062, 820380; Praia Brava, Parati (RJ) - 1 exemplar - 760141.

\section{Ordem Gasterosteiformes}

\section{Familia Fistulariidae}

26.1. Fistularia Linnaeus, 1758

26.1.1. Fistularia petimba Lacépède, 1803

Nome vulgar: trombeta.

Distribuição conhecida: no Atlântico tropical.

Material colecionado: Arraial do Cabo (RJ) - 1 exemplar - 840707.

O exemplar acima citado foi encontrado morto na praia.

\subsubsection{Fistularia tabacaria Linnaeus, 1758}

Nome vulgar: trombeta.

Distribuição conhecida: dos EUA (Nova Inglaterra) à Garopaba (SC), sendo que Leim \& Scott in Fritzsche (1976) citam a ocorrência ocasional de jovens na Nova Escócia (Canadá).

Material colecionado: Praia de Guanabara, Ilha do Governador, Baía de Guanabara (RJ) - 2 exemplares - 860824, 860837.

27. Familia Syngnathidae

27.1. Hippocampus Rafinesque, 1810 27.1.1. Hippocampus erectus Perry, 1810

Nome vulgar: cavalo-marinho. 
Distribuição conhecida: do Canadá (Nova Escócia) à Argentina .

Material colecionado: $22^{\circ} 25^{\prime}$ lat. S, $41^{\circ} 43^{\prime}$ long. W, Ilha do Santana (RJ) - 3 exemplares - $840579 ; 23^{\circ} 12^{\prime}$ lat. S, $44^{\circ} 07^{\prime}$ long. W - $23^{\circ} 13^{\prime}$ lat. S, $44^{\circ} 11^{\prime}$ long. W, Ilha Grande (RJ) - 2 exemplares - 770293.

27.1.2. Hippocampus reidi Ginsburg, 1933

Nome vulgar: cavalo-marinho.

Distribuição conhecida: nas Bermudas, Caribe e do Recife (PE) ao Rio Grande do Sul, BR.

Material colecionado: Guarapari (ES) - 1 exemplar - 820370; Praia de Guanabara, Ilha do Governador, Baila de Guanabara (RJ) - 1 exemplar - 860838; Praia da Urca, Baía de Guanabara (RJ) - 2 exemplares - 770187, 840683.

27.2. Oostethus Hubbs, 1929

27.2.1. Oostethus lineatus (Kaup, 1856)

Nome vulgar: peixe-cachimbo.

Distribuição conhecida: dos EUA (Carolina do Norte) ao sul do Brasil.

Material colecionado: Rio Bananal, Maricá (RJ) - 1 exemplar - 820366.

Dawson (1979), mesmo preferindo considerar lineatus como uma subespécie de 0 . brachyurus (Bleeker, 1853), ressalta que a maior parte dos autores recentes vem adotando o nome $O$. lineatus (Kaup, 1856). Este é o caso, por exemplo, de FIGUEIREDO \& MENEZES (1980), cujo critério foi adotado na identificação do exemplar acima citado.

27.3. Syngnathus Linnaeus, 1758

27.3.1. Syngnathus pelagicus Linnaeus, 1758

Nome vulgar: peixe-cachimbo.

Distribuição conhecida: Das Bermudas à Argentina .

Material colecionado: Praia da Guanabara, Ilha do Governador, Baia de Guanabara (RJ) - 6 exemplares - 840656, 860484, 860875 .

\section{Ordem Scorpaeniformes}

\subsection{Scorpaena Linnaeus, 1758}

27.4.1. Scorpaena brasiliensis Cuvier, 1829

Nome vulgar: beatinha, mangangá .

Distribuição conhecida: dos EUA (Virgínia) à Garopaba (SC).

Material colecionado: Ilha Rasa (RJ) - 1 exemplar - 840575; Do meio da Baila de Guanabara (RJ) - 1 exemplar - 870886; Praia de Guanabara, Ilha do Governador, Baía de Guanabara (RJ) - 1 exemplar - 860873; Ilhas Tijucas (RJ) - 1 exemplar 760180.

\subsubsection{Scorpaena isthmensis Meek \& Hildebrand, 1928}

Distribuição conhecida: dos EUA (Carolina do Norte) a Santos (SP).

Material colecionado: Ilha Rasa (RJ) - 1 exemplar - 840573; Do meio da Baía de Guanabara (RJ) - 1 exemplar - 840654; Praia de Guanabara, Ilha do Governador, Baía de Guanabara (RJ) - 1 exemplar - 840599; Saco da Piraquara de Fora, Angra dos Reis (RJ) - 1 exemplar - 830474; Caieira da Barra Sul, Florianópolis (SC) - 1 exemplar - 770226. O limite sul da distribuição desta espécie foi fornecido por Figueiredo (comunicação pessoal).

O exemplar 770226 representa uma extensão da distribuição da espécie para o sul. 
27.4.3. Scorpaena plumieri Bloch, 1789

Nome vulgar: beatinha, mangangá.

Distribuição conhecida: dos EUA (Massachusetts) à Florianópolis (SC).

Material colecionado: Meaípe, Guarapari (ES) - 1 exemplar - 770231; Praia da Urca, Baĭa de Guanabara (RJ) - 2 exemplares - 840572, 870924; Saco da Piraquara de Fora, Angra dos Reis (RJ) - 1 exemplar - 830498.

\section{Familia Triglidae}

29.1. Prionotus Lacépède, 1801

29.1.1. Prionotus punctatus (Bloch, 1797)

Nome vulgar: cabrinha.

Distribuição conhecida: na Jamaica e de Belize à Argentina.

Material colecionado: Meaĭpe de Guarapari (ES) - 1 exemplar - 760065; Baĭa de Guanabara (RJ) - 1 exemplar - 860795; Do meio da Baía de Guanabara (RJ) 2 exemplares - 840639; Praia da Guanabara, Ilha do Governador, Baía de Guanabara (RJ) - 1 exemplar - 860834; Praia da Engenhoca, Ilha do Governador, Baĭa de Guanabara (RJ) - 1 exemplar - 870884; Sepetiba, Rio de Janeiro (RJ) 1 exemplar - 830407; Ilha Grande (RJ) - 6 exemplares - 770303; $23^{\circ} 12^{\prime}$ lat. S, $44^{\circ} 07^{\prime}$ long. W - $23^{\circ} 13^{\prime}$ lat. S, $44^{\circ} 11^{\prime}$ 'long. W, Ilha Grande (RJ) - 1 exemplar 770289; Saco da Piraquara de Fora, Angra dos Reis (RJ) - 9 exemplares $830433,830504,830519,830524,830530,830543$; Base Oceanográfica Norte da Universidade de São Paulo, Ubatuba (SP) - 3 exemplares - 790344.

O exemplar 870884 foi encontrado morto, boiando.

\section{Ordem Dactylopteriformes}

\section{Familia Dactylopteridae}

30.1. Dactylopterus Lacépède, 1801

30.1.1.Dactylopterus volitans (Linnaeus, 1758)

Nome vulgar: cabra-bode, coió, voador.

Distribuição conhecida: do Canadá (Nova Escócia) à Argentina.

Material colecionado: Arraial do Cabo (RJ) - 1 exemplar - 770251; Praia de Itaipu, Niterói (RJ) - 2 exemplares - 760151; Praia Vermelha, Baía de Guanabara (RJ) - 1 exemplar - 840592; Praia da Urca, Baĭa de Guanabara (RJ) - 4 exemplares - $840617 ; 23^{\circ} 12^{\prime}$ lat. S, $44^{\circ} 07^{\prime}$ long. W $-23^{\circ} 13^{\prime}$ lat. S, $44^{\circ} 11^{\prime}$ long. W, Ilha Grande (RJ) - 2 exemplares - 770276; Em águas adjacentes à Central Nuclear Almirante Álvaro Alberto, Angra dos Reis (RJ) - 2 exemplares - 830469; Saco da Piraquara de Fora, Angra dos Reis (RJ) - 3 exemplares - 830432, 830483 .

\section{AGRADECIMENTOS}

Ao Professor Mario Jorge Ignacio Brum, responsável pelo Laboratório de Ictiologia da Universidade Federal do Rio de Janeiro, por permitir a realização deste trabalho ao apoiá-lo e facilitar o acesso ao material depositado nesta coleção. 
Ao Doutor José Lima de Figueiredo, do Museu de Zoologia da Universidade de São Paulo, pelo apoio e esclarecimentos de várias dúvidas surgidas no decorrer deste trabalho.

Ao Biólogo Paulo Ricardo Schwingel, da Fundação Universidade do Rio Grande, pela identificação de Psammobatis glansdissimilis.

Ao Professor Marcos Venício Mazzetti, da Faculdade Maria Thereza (Niterói, RJ), pela identificação das espécies dos gêneros Anchoa e Engraulis.

Ao Professor Ulisses Leite Gomes, da Universidade do Estado do Rio de Janeiro, pelo apoio e auxilio na identificação do exemplar do gênero Psammobatis.

Ao Doutor Victor Sadowsky, do Instituto de Pesca (Santos, SP), pelos esclarecimentos quanto ao limite sul de distribuição geográfica de Gymnura micrura no Atlântico Ocidental.

Às Biólogas Maria Leonisa Sanchez Nuñez e Helena São Thiago, da Universidade Federal do Rio de Janeiro, pelas críticas e sugestões apresentadas durante a elaboração deste trabalho.

A Bióloga Cynthia Lara de Castro Manso, da Universidade Federal do Rio de Janeiro, pelo auxilio na correta interpretação de mapas do município de Angra dos Reis e pelas sugestões apresentadas.

Ao Biólogo Charles Frederic de Meirelles Lagden Muratori, da Universidade Federal do Rio de Janeiro, por permitir a inclusão de dados da sua monografia de conclusão de cursos (que se refere à maior parte do material coletado na Baía de Guanabara).

\section{BIBLIOGRAFIA CONSULTADA}

BENVEGNÚ, G. Q., 1973. Relação dos peixes teleósteos, in Relatório sobre a segunda pesquisa oceanográfica e pesqueira do Atlântico sul entre Torres e Maldonado (lat. $29^{\circ} \mathrm{S}$ $35^{\circ}$ S). Programa Rio Grande do Sul - II. Publção esp. Inst. oceanogr. S. Paulo, São Paulo, (3): 489-500.

BOHLKE, J. E., 1978. Muraenidae, in W. Fischer q.v.

BOHLKE, J. E., 1978. Ophichthidae, in W. Fischer q.v.

BRADBURY, M. G. 1978. Ogcocephalidae, in W. Fischer q.v.

CARUSO, J. H., 1978. Lophiidae, in W. Fischer, q. v.

CERVIGON, F. \& BASTIDA, R., 1974. Contribución al conocimiento de la fauna ictiologica de la Província de Buenos Aires (Argentina). An. Soc. Cient. Argent. 192 (1-3): 3-20.

COHEN, D. M., 1978. Gadidae, in W. Fischer q. v.

COHEN, D. M., 1978. Ophidiidae, in W. Fischer q. v.

COHEN, D. M. \& J. C. NIELSEN, 1978. Guide to the identification of genera of the fish Order Ophidiiformes with a tentative classification of the order. NOAA Tech. Rep., NMFS Circ. 417, 72 p. 
COLLETTE, B. B., 1978. Batrachoididae, in W. Fischer q.v.

COLlETTE, B. B., 1978. Belonidae, in W. Fischer q. v.

COLLETTE, B. B., 1978. Hemiramphidae, in W. Fischer q.v.

DAWSON, C. E., 1979. Review of the polytypic doryhamphine pipefish Oostethus brachyurus (Bleeker). Bull. mar. Sci. 29 (4): 465-480.

ESCHMEYER, W. N., 1969. A systematic review of the scorpionfishes of the Atlantic Ocean. Occ. Pap. Calif. Acad. Sci. 79: iv + 143 p.

Figueiredo, J. L., 1977. Manual de Peixes Marinhos do Sudeste do Brasil. I. In trodução. Cações, Raias e Quimeras; 104 pp. Museu de Zoologia da Universidade de São Paulo, São Paulo.

FIGUEIREDO, J. L., 1981. Estudo das Distribuições Endêmicas de Peixes da Província Zoogeográfica Marinha Argentina, $121 \mathrm{p}$. Tese de doutoramento. Universidade de São Paulo, São Paulo.

FIGUEIREDO, J. L. \& N. A. MENEZES., 1978. Manual de Peixes Marinhos do Sudeste do Brasil. II. Teleostei (1), 110 p. Museu de Zoologia da Universidade de São Paulo, São Paulo.

Figueiredo, J. L. \& N. A. MENEZES, 1980. Manual de Peixes Marinhos do Sudeste do Brasil. III. Teleostei (2), 90 p. Museu de Zoologia da Universidade de São Paulo, São Paulo.

FISCHER, W, (Ed.). 1978, FAO Species Identification Sheets for Fishery Purpose. Western Central Atlantic (Fishing Area 31). Vols, 1-7. Roma, FAO, pag. var.

FRITZSCHE, R. A., 1976. A review of the cornetfishes, genus Fistularia (Fistulariidae), with a discussion of intrageneric relationships and zoogeography. Bull. mar. Sci. 26 (2): 196-204.

FRITZSCHE, R. A., 1978. Fistulariidae, in W. Fischer q.v.

HABURAY, K., R. W. HASTINGS, D. DeVRIES \& J. MASSEY, 1974. Tropical marine fishes from Pensacola, Florida. Fla. Sci. 37 (2):105-109.

HASTINGS, P. A. \& S. A. BORTONE., 1976. Additional notes on tropical marine fishes in the northern Gulf of Mexico. Fla. Sci. 39 (2): 122-125.

HIGUCHI, H., E. G. REIS \& F. G. ARAÚJO, 1982. Uma nova espécie de bagre marinho do litoral do Rio Grande do Sul e considerações sobre o gênero nominal Netuma Bleeker, 1858 no Atlântico sul ocidental (Siluriformes, Ariidae). Atlântica, Rio Grande, 5: 1-15.

LEMA, T., 1976. Ocorrência de várias espécies de peixes tropicais marinhos na costa do Estado de Santa Catarina, Brasil (Osteichthyes, Actinopterygii, Teleostei). Iheringia, Sér. Zool., Porto Alegre (49): 39-65.

LEMA, T.; C.A.S. LUCENA \& Z. M.S. LUCENA, 1980. Novas adendas à ictiofauna marinha do ex tremo sul do Brasil. Iheringia, Sér. Zool., Porto Alegre, (56): 103-120.

LUCENA, C. A. S. \& Z. M. S. LUCENA, 1981. Catálogo dos peixes marinhos do Mu seu de Ciências da Pontifícia Universidade Católica do Rio Grande do Sul. Elasmobranchiomorphi, Teleostomi ( $1^{\text {a }}$. parte). Comun. Mus. Ci. PUCRGS, Por to Alegre, (21): 1-66. 
MARKLE, D. F., W. B. SCOTT \& A.C. KOHLER, 1980. New and rare records of Canadian fishes and the influence of hydrography on resident and nonresident Scotian Shelf ichthyofauna. Can. J. Fish. Aquat. Sci. 37:49-65.

McEACHRAN, J. D., 1983. Results of the research cruises of FRV "Walther Herwig" to South American. LXI. Revision of the South American skate genus Psammobatis Günther, 1870 (Elasmobranchii: Rajiformes, Rajidae). Arch. Fischereiwiss., Berlin, 34: 23-80.

McEACHRAN, J. D. \& W. N. ESCHMEYER, 1973. Range ex tension for the scorpionfish Scorpaena isthmensis. Fla. Sci. 36 (2-4): 209-211.

MENNI, R. C. \& A.M. MIQUELARENA, 1976. Sobre dos especies argentinos de Batrachoididae (Pisces, Batrachoidiformes). Physis, B. Aires (A) 35 (91): 205-219.

MILLER, G. C. \& W. RICHARDS, 1978. Triglidae, in W. Fischer q. v.

MILSTEIN, C. B. \& D. L. THOMAS, 1976. Fishes new or uncommon to the New Jersey coast. Chesapeake Sci. 17(3): 198-204.

NONATO, E. F., A. C. Z. Amaral \& J. L. FIGUEIREDO, 1983, Contribuição ao conhecimento da fauna de peixes do litoral norte do Estado de São Paulo. Bolm Inst.oceanogr., S. Paulo, 32 (2): 143-152.

PERRONE, E. C., J. L. HELMER \& C. ZAMPROGNO, 1985. Peixes anguilliformes do litoral do Espirito Santo. Resumos, XII Congresso Brasileiro de Zoologia: 169.

PIETSCH, T. W., 1978. Antennariidae, in W. Fischer q. v.

RANDALL, J. E., 1978. Congridae, in W. Fischer q. v.

RANDALL, J. E., 1978. Synodontidae, in W. Fischer q.v.

SADOWSKY, V., 1973. Relação dos peixes cartilaginosos, in Relatório sobre a segunda pesquisa oceanográfica e pesqueira do Atlântico sul entre Torres e Maldonado (lat. $29^{\circ} \mathrm{S}$ $\left.35^{\circ} \mathrm{S}\right)$. Programa Rio Grande do Sul - II. Publção esp. Inst. oceanogr. S. Paulo, São Paulo, (3): $483-488$.

SMITH-VANIZ, W. F., 1978. Dactylopteridae, in W. Fischer q.v.

TAYLOR, W. R. \& N. A. MENEZES, 1978. Ariidae, in W. Fischer q.v.

VERGARA RODRIGUEZ, R., 1978. Merlucciiidae, in W. Fischer q.v .

WHITEHEAD, P. J. P., 1978. Clupeidae, in W. Fischer q.v.

WHITEHEAD, P. J.P., 1978. Engraulidae, in W. Fischer q.v.

WHITEHEAD, P. J. P. \& Vergara Rodríguez, R., 1978. Elopidae, in W. Fischer q. v.

WOODS, L. P. \& D. W. GREENFIELD, 1978. Holocentridae, in W. Fischer q.v. 